\title{
Optimal Sequencing of Anti-HER2 Therapy
}

\author{
Chair: Hans-Joachim Lück ${ }^{a}$ \\ Participants: Michael Untch ${ }^{\mathrm{b}}$ Christian Jackisch ${ }^{\mathrm{c}}$ Christoph Zielinski ${ }^{\mathrm{d}}$ Rupert Bartsch ${ }^{\mathrm{d}}$ \\ aGynäkologisch-Onkologische Schwerpunktpraxis Hannover, \\ ${ }^{\mathrm{b} B r u s t z e n t r u m / F r a u e n k l i n i k, ~ H E L I O S ~ K l i n i k u m ~ B e r l i n-B u c h, ~}$ \\ cKlinik für Gynäkologie und Geburtshilfe, Klinikum Offenbach, Germany \\ dKlinik für Innere Medizin I, Universitätskliniken der Medizinischen Universität Wien, Austria
}

\section{Question 1: Could the Time Interval from the End of Adjuvant Trastuzumab Therapy to Onset of Metastasis (<1 Year vs. $>1$ Years) be Relevant for the Selection of HER2-Directed Therapy?}

Untch: There is no clear-cut definition of trastuzumab resistance or sensitivity. First, because patients who received trastuzumab in the adjuvant setting also received adjuvant chemotherapy, mostly anthracycline and taxane containing regimens. Second, because probably there is a biological difference between patients with hormone receptor-positive tumors compared to patients with hormone receptor-negative tumors. Therefore, in patients with hormone receptor-negative, HER2-overexpressing tumors probably the definition of trastuzumab resistance is adequate if the patient develops recurrence and/or metastases within 1 year after completion of adjuvant trastuzumab therapy.

For patients who have hormone receptor-positive, HER2overexpressing tumors, this definition might be too tight, therefore my proposal is to define trastuzumab resistance for patients who have recurrence and/or metastases within 24 months after completion of trastuzumab therapy. For patients with partial or complete trastuzumab resistance, re-initiation with trastuzumab might not be the best first choice; for these patients a treatment sequence for metastases might be best. For patients with partial sensitivity to trastuzumab (recurrence of metastases more than 1 year after completion of trastuzumab in patients with hormone receptor-negative tumors or more than 24 months after completion of adjuvant trastuzumab in patients with hormone receptor positive tumors), the best choice might be either docetaxel with trastuzumab plus pertuzumab for hormone receptor-negative patients or the combination of lapatinib plus an aromatase inhibitor for patients with hormone receptor-positive tumors.

Jackisch: At this time this is a kind of historical question. The disease free interval of less or more than 1 year is a rele- vant prognostic information about the aggressiveness of the given tumor type in HER2-overexpressing disease. From a clinical point of view we all agree on the fact that HER2 overexpression is the key driver of a negative prognosis. Thus, in any case I would opt for ongoing blockade of this pathway using HER2 directed therapy in the metastatic setting.

Bartsch/Zielinski: Only a very limited number of patients in the CLEOPATRA trial had received prior adjuvant therapy with trastuzumab. Furthermore, the minimum diseasefree interval requested for inclusion was 12 months. This was reduced to 6 months in the ongoing PERUSE study (NCT01572038), but results of that trial are pending. Therefore, for the time being, the only data concerning the activity of third generation anti-HER2 agents in early relapsers are derived from the EMILIA study of T-DM1. T-DM1 was superior to lapatinib plus capecitabine in all subgroups, and patients included into the lapatinib plus trastuzumab trial were heavily pretreated [Blackwell KL et al. J Clin Oncol 2012;30:2585-2592]. Therefore, in our opinion, T-DM1 is the agent of choice in those rare patients with very early relapse after adjuvant trastuzumab.

\section{Question 2: Does the Hormone Receptor Status Have an Impact on the Choice of HER2-Directed Therapy in the Metastatic Setting?}

Untch: Please also see my comments to the first question. We have learned that hormone receptor-positive patients behave different from hormone receptor-negative patients in the presence of HER2-overexpression. Therefore, in the metastatic setting the first therapeutic choice, especially for patients with aggressive or symptomatic disease and for patients with hormone receptor-negative tumors, is obviously the combination of an anti-HER2 agent with chemotherapy, otherwise in patients with low tumor burden, oligometatastic

\section{KARGER \\ Fax +497614520714 \\ Information@Karger.com}

www.karger.com (c) 2014 S. Karger GmbH, Freiburg

$1661-3791 / 14 / 0092-0138 \$ 39.50 / 0$

Accessible online at:

www.karger.com/brc
Prof. Dr. med. Hans-Joachim Lück

Gynäkologisch-Onkologische Schwerpunktpraxis Hannover

Pelikanplatz 23, 30177 Hannover, Germany

hlueck@goph.de 
disease, or less clinical symptoms a good choice might be the combination of an anti-HER2 agent with aromatase inhibitors.

Jackisch: Another important piece of information. Even if this is a small subset of tumors presenting as dual or triple positive exposing positive measures of estrogen and/or progesterone hormone receptors, this is relevant information for my treatment decision. In a postmenopausal patient presenting with this tumor profile I am considering in the metastatic setting to go for a horizontal dual blockade using an aromatase inhibitor along with either trastuzumab or lapatinib in order to avoid the use of a combination with chemotherapy and one of the anti-HER2 agents. These are often scenarios where I do not need a chemotherapy regimen in the first place in order to control the disease at that time. In this case it is very important that signs and symptoms of the disease are under control and the patient is not symptomatic. Even in visceral metastases with low progression profile this might be a good option.

Bartsch/Zielinski: The hormone receptor status is of some relevance in HER2-positive disease although most often it will not influence treatment decisions. In the neoadjuvant setting, it is a negative predictor of pathological complete remission ( $\mathrm{pCR}$ ) but a positive predictor of outcome in patients without $\mathrm{pCR}$. In the metastatic setting, there is the option of first-line combination therapy with an aromatase-inhibitor plus a HER2 targeted agent in patients with low-risk metastatic disease or contraindications to chemotherapy. No survival benefit, however, was shown with this combination over first-line endocrine treatment alone; therefore, this strategy is an option limited to a highly selected population.

Recently, the combination of trastuzumab plus lapatinib was approved by EMA for HER2-positive, hormone receptor-negative patients, as this population derived the largest relative benefit [Blackwell et al. J Clin Oncol 2012;30:25852492]. For the time being, this combination would be the only one where we would feel that the hormone receptor status might definitely influence treatment decisions.

\section{Question 3: What Would Be the Most Likely Treatment Option for Patients Who Received a Trastuzumab-Pertuzumab Combination, or a Trastuzumab-Lapatinib Combination in the Adjuvant Setting?}

Untch: There are no data on how to treat patients with this type of disease. From the extrapolation of data from patients with metastatic disease the best choice is probably T-DM1.

Jackisch: These days we see patients progressing after a dual blockade. As we know the combination of trastuzumab and pertuzumab was introduced together with docetaxel in the CLEOPATRA trial in the metastatic setting on almost trastuzumab naïve patients. This combination might be used in Germany in the framework of a clinical trial in the neoadjuvant setting. In contrast to this the vertical dual blockade introducing trastuzumab and lapatinib is an interesting chemotherapy free combination that has been introduced in the later treatment lines of metastatic breast cancer. Thus, this combination is currently more unlikely to be used in the adjuvant setting. If the disease is progressing in the adjuvant setting while under the treatment option of horizontal dual blockade using trastuzumab and pertuzumab the option of the vertical dual blockade might be an option if the patient refuses another chemotherapy regimen. In addition to these options we learned from the EMILIA trial that the introduction of T-DM1 is a very promising option in these two scenarios. T-DM1 is very active in patients progressing after trastuzumab based regimens providing a substantial survival benefit. This would be my first choice after progression on trastuzumab and pertuzumab in the metastatic setting.

Bartsch/Zielinski: We believe that a relatively short-course dual inhibition of HER2 in the neoadjuvant setting would not alter the current treatment algorithm after full-course adjuvant therapy with trastuzumab. Of course, we would assume different mechanisms of resistance in patients progressing after 1 year of trastuzumab, trastuzumab plus pertuzumab, or trastuzumab plus lapatinib. Currently, we have no knowledge from large prospective trials with regards to the optimal treatment approach in such individuals and any answer would be pure speculation. Still, we would feel that in patients progressing upon trastuzumab plus pertuzumab, first-line therapy with T-DM1 or lapatinib plus trastuzumab might be an option, in patients progressing upon trastuzumab plus lapatinib, T-DM1 or trastuzumab plus pertuzumab might be advisable.

\section{Question 4: Is T-DM1 for Primary Treatment Failure of Trastzumab-Pertuzumab the First Option, or Could Lapatinib Containing Regimens Be More Appropriate?}

Untch: Both possibilities have to be discussed with the patients, including the expected side effects. I personally tend to opt for T-DM1 since the antibody has shown superior effects in the direct comparison with lapatinib/capecitabine in the EMILIA study. But these patients were not pretreated with trastuzumab/pertuzumab, therefore the option of lapatinib with trastuzumab might also be a valid approach.

Jackisch: Since the data of the EMILIA trial are available, this is my first choice in patients progressing on these both options of a dual blockade of the HER2 receptor pathway. Using T-DM1 in the metastatic setting provides us with a very 
rapid onset of remission or controlling the signs and symptoms of the disease with little toxicity. We have to monitor cardiac function as well as thrombocytopenia. My patients appreciate this treatment option offering them a new quality of life. The vertical dual blockade with lapatinib and trastuzumab is another interesting alternative, which is second after T-DM1 in my practice. It might be interesting to know that trastuzumab is available in Germany as subcutaneous (sc) formulation. In this setting we have the option to use trastuzumab sc together with the oral application of lapatinb enhancing the quality of life in a different way allowing them to enjoy the benefit of the short infusion duration of trastuzumab sc.

Bartsch/Zielinski: Again, this is a highly relevant question without a clear answer. After failure of a dual targeting approach, T-DM1 (and its alternative mechanism of action) may be interesting. Clearly, however, we cannot rule out a relevant activity of lapatinib plus trastuzumab in this patient subgroup. A treatment strategy of pertuzumab beyond progression might also be interesting, but we have no clinical data supporting this approach so far. Finally, results from the MARIANNE trial (NCT01120184) might render the question obsolete if the combination of T-DM1 plus pertuzumab becomes a novel treatment standard in the first-line setting.

\section{Question 5: Could It Make Sense to Use Lapatinib for Re-Expression of HER2 Receptors?}

Untch: There are no clear clinical data to prove that lapatinib can re-express HER2 receptors. Therefore, our approach, which is also a part of our national guideline for the treatment of patients with breast cancer, is to try to get histology from the metastatic site (like liver metastases, pleural effusion, ascites, lymph node biopsy, skin biopsy, etc.) as often as possible and if the metastases or recurrent disease show HER2 overexpression, then obviously an anti-HER2 treatment should be state of the art for the patient, taking into account the clinical situation, symptoms, previous treatment, therapeutic index, patient expectations, etc.

Jackisch: Again I consider lapatinib a very active and interesting drug in the treatment of HER2-positive disease. This might be used in combination with trastuzumab or a variety of other cytotoxic drugs in the late lines of treatment of metastatic disease and, as outlined above, in combination with letrozole a third-generation aromatase inhibitor. Lapatinib is known for this mode of action, resensitizing HER2-positive disease to this type of treatment.

Bartsch/Zielinski: Scaltriti et al. provided us with fascinating data suggesting that lapatinib inhibits HER2 downregulation in response to trastuzumab therapy [Scaltriti $\mathrm{M}$ et al. Oncogene 2009;28:803-814]. If HER2 downregulation is indeed a relevant mechanism of resistance to trastuzumabbased therapy, the combination of lapatinib plus trastuzumab might reverse this effect. Indeed, we believe that the positive results of the Blackwell study leading to the approval of trastuzumab plus lapatinib in HER2-positive / hormone receptornegative patients are partly caused by this effect.

\section{Participants}

Prof. Dr. med. Christian Jackisch

Klinik für Gynäkologie und Geburtshilfe

Klinikum Offenbach

Starkenburgring 66

63069 Offenbach, Germany

Christian.Jackisch@Sana.de

Prof. Dr. med. Michael Untch

Frauenklinik / Interdisziplinäres Brustzentrum

HELIOS Klinikum Berlin-Buch

Schwanebecker Chaussee 50, 13125 Berlin, Germany

michael.untch@helios-kliniken.de

PD Dr. Rupert Bartsch

Comprehensive Cancer Center

Klinische Abteilung für Onkologie

Universitätsklinik für Innere Medizin 1

Medizinische Universität Wien

Währinger Gürtel 18-20, 1090 Wien, Austria

rupert.bartsch@meduniwien.ac.at

Univ.-Prof. Dr. Christoph Zielinski

Universitätskliniken und Krebszentrum

der Medizinischen Universität Wien

Klinik für Innere Medizin I

Klinische Abteilung für Onkologie

Währinger Gürtel 18-20

1090 Wien, Austria

christoph.zielinski@meduniwien.ac.at 